\title{
Análise morfométrica de populações do caranguejo-uçá (Ucides Cordatus L.) (Crustacea - Decapoda) em manguezais do litoral do Espírito Santo ${ }^{1}$
}

\author{
Cristiana Libardi Miranda ${ }^{2}$ \\ Renata Pasini Barbosa ${ }^{2}$ \\ Andressa de Sousa Lima ${ }^{2}$ \\ Júlio Cola Rovida ${ }^{2}$ \\ Jaqueline Coelho Rocha ${ }^{2}$ \\ Leticia Soncini de Souza ${ }^{2}$ \\ Aldrin Vieira Pires ${ }^{3}$ \\ Rodrigo Lemes Martins ${ }^{4}$ \\ Samuel Rezende Paiva ${ }^{5}$
}

\begin{abstract}
Resumo
O caranguejo-uçá (Ucides cordatus) é um crustáceo (Decápoda: Brachyura) de grande valor cultural, biológico e comercial em todo Espírito Santo. Contudo, ainda há uma grande lacuna de conhecimentos acerca de sua biologia. Com o intuito de minimizar essa carência, o presente trabalho realizou um estudo sobre a caracterização morfológica de três populaçôes do caranguejo-uçá (Ucides cordatus L.) em manguezais do Espírito Santo. Foram analisadas quatro características morfométricas em 172 indivíduos machos, coletados nas cidades de São Mateus (N=62), Vitória (N=60) e Anchieta (50). Foi observada diferença significativa entre as três populaçôes $(\mathrm{p}<0,01)$ por meio de uma Análise de Variância Multivariada. O maior grau de similaridade foi encontrado entre as populaçôes de São Mateus (Norte) e Anchieta (Sul), de modo que a população de Vitória (Centro) foi agrupada separadamente, evidenciando-se divergência entre essa população e as demais. Os resultados sugerem pressões de seleção diferentes nas populaçôes do interior em relação à população da capital, o que pode estar relacionado a uma maior ação antrópica nas populações da capital.
\end{abstract}

Palavras-chave: $U$. cordatus - divergência genética; conservação animal; MANOVA.

\section{INTRODUÇÃO}

O manguezal é um ecossistema costeiro de transição entre a terra e o mar, considerado um dos ecossistemas marinhos mais produtivos do planeta. O mangue é encontrado nas regiōes tro-

\footnotetext{
Financiamento FAESA/FACITEC. Vitória-ES.

${ }^{2}$ Biólogo. FAESA. Vitória-ES.

${ }^{3}$ DS em Genética e Melhoramento Animal. FAESA. Vitória-ES.

${ }^{4}$ MS em Ecologia. FAESA. Vitória-ES.

${ }^{5}$ MS em Genética e Melhoramento Animal. FAESA. Vitória-ES.

Correspondência para / Correspondence to:

Cristiana Libardi Miranda

R. Brasília, 277- Bairro Itapemirim.

29.149-000 Cariacica-Espírito Santo-Brasil.

Tel: (27)3336-0372.

E-mail: clibardihd@terra.com.br
} 
picais e subtropicais, entre os trópicos de Câncer e de Capricórnio, funcionando como verdadeiros berçários do mar, locais onde se desenvolvem e se reproduzem inúmeras espécies marinhas e estuarinas (NUNES, 1998).

Recentemente, uma revisão sobre o manejo e a sustentabilidade econômica em áreas de manguezais brasileiros conclui que a pesca de crustáceos é a que fornece maiores retornos financeiros para populações tradicionais (KJERFET; LACERDA apud FREIRE, 1998).

O caranguejo-uçá (Ucides cordatus Linnaeus, 1763) é uma das espécies mais significativas da fauna do ecossistema manguezal, constituindo um dos principais recursos pesqueiros dos manguezais do Estado do Espírito Santo, em função da larga aceitação para consumo (CARMO, 1987). Essa espécie, apesar de atingir grande porte na fase adulta, apresenta uma taxa de crescimento extremamente reduzida (DIELE, 2000). Levando em consideração a pequena taxa de crescimento, a espécie vem sendo constantemente agredida por ações antrópicas, que incluem a coleta indiscriminada, inclusive nos períodos em que é proibida, e pela poluição, com o lançamento de esgotos e demais dejetos industriais e domésticos. Essas ações ocasionam uma redução na população do $U$. cordatus e no tamanho do caranguejo capturado, levando os órgãos gestores a intensificar a fiscalização para a proteção desse recurso pesqueiro (PINHEIRO; FISCARELLI, 2001).

Estudos sobre a biologia e o ciclo de vida do caranguejo-uçá vêm sendo intensificados, servindo de subsídio para a conservação dessa espécie. Alcantra-Filho (1982) estudou a estrutura populacional, a reprodução, o dimorfismo sexual, a diversificação intraespecífica e a distribuição espacial do caranguejo-uçá nos manguezais dos Rios Pará (Pará) e Pomonga (Sergipe). Encontram-se também estudos da dispersão larval (FREIRE, 1998) e do desenvolvimento pós-embrionário (RODRIGUES, 1982).

Em razão desses fatores e da grande ação antrópica nos manguezais do Espírito Santo, fazem-se necessários estudos que possibilitem avaliar a divergência genética do caranguejo-uçá, relacionando-a com parâmetros morfométricos de importância comercial. Considerando-se alterações morfológicas e na freqüência gênica, é possível avaliar o efeito da coleta sobre essas freqüências, ou mesmo evidenciar a relação das freqüências em função de outra variável, como poluição e degradação do meio ambiente.

A variabilidade genética, também chamada de biodiversidade molecular, pode ser usada como instrumento de investigação por ecólogos e sistematas em diversos ramos, como, por exemplo: verificar as afinidades e os limites entre as espécies; detectar modos de reprodução e estrutura familiar; estimar níveis de migração e de dispersão nas populaçōes (MATIOLI, 2001).

A variabilidade nos permite comparar indivíduos, populações ou espécies diferentes. Ao estudar a variação genética em uma população, devemos considerar, além da deriva genética, as outras forças que possam estar envolvidas nas alteraçōes das frequiências alélicas, ou seja, mutações, migraçōes, eventos de seleção e suas combinações (MATIOLI, 2001).

As pequenas diferenças entre indivíduos, como tamanho do corpo ou número de ovos, são variaçōes evidentes, chamadas de variação contínua ou variação quantitativa (FUTUYMA, 2002). Essas variaçōes fenotípicas interferem nas estimativas das freqüências alélicas baseadas em morfometria (PAIVA, 2001). O estudo fenotípico é o ponto de partida para qualquer análise genética, visto que um único genótipo pode produzir fenótipos diferentes, bem como o mesmo fenótipo pode ser produzido por genótipos diferentes, dependendo do ambiente no qual o indivíduo se desenvolveu (GRIFFTHIS et al., 2002).

Estudos de variação gênica têm sido empregados para fins de conservação, como, por exemplo, em psitacídeos brasileiros (TAVARES; MIYAKI, 2003), boto rosa (VIANNA; MARMONTEL; SANTOS, 2003) e lobo-guará (PRATES-JR et al., 2003). Esses estudos foram realizados com base em dados moleculares e mostraram-se bastante eficientes sob esse aspecto, servindo de subsídio para a conservação dessas espécies.

Paiva (2001) utilizou-se de dados morfológicos como auxílio para a identificação 
de divergência genética em populaçôes de Styanax bimaculalus (lambari-rabo-amarelo), apresentando-se extremamente importante para o estudo em questão. Fonseca e colaboradores (2000) realizaram estudos de divergência genética entre raças suínas; Pires e colaboradores (2002) estudaram a divergência genética entre seis linhas de aves; e Carneiro e colaboradores (2002) estudaram a divergência genética entre quatro linhagens de matrizes de frango de corte.

Os métodos estatísticos apropriados para esses estudos, em que várias variáveis devem ser consideradas simultaneamente, são as técnicas de análise de variância multivariada (MANOVA). As técnicas de análise multivariada combinam as múltiplas informações provenientes de uma unidade experimental, obtendo-se informaçōes que não podem ser serem obtidas com o uso da análise univariada (ARAÚJO, 1995).

Existem muitas ferramentas de análise multivariada. Uma delas, que se baseia na técnica de variáveis canônicas, foi relatada por Rao em 1952. Trata-se de um processo para a avaliação do grau de similaridade genética entre progenitores, que leva em consideração tanto a matriz de covariância residual quanto a de covariância fenotípica entre os caracteres avaliados (CRUZ; REGAZZI, 1997).

Devido à grande importância dessa espécie, este estudo tem por objetivo determinar, por meio de análise multivariada das medidas morfométricas, a existência de divergências fenotípicas entre populações de $U$. cordatus dos municípios de São Mateus, Vitória e Anchieta, litoral norte, centro e sul do Espírito Santo, respectivamente.

\section{MATERIAS E MÉTODOS}

As coletas foram realizadas nos manguezais dos municípios de São Mateus $\left(18^{\circ} 42^{\prime} 58^{\prime \prime} \mathrm{S}\right.$ e $\left.39^{\circ} 51^{\prime} 32^{\prime \prime W}\right), \quad$ Vitória $\left(20^{\circ} 19^{\prime} 10^{\prime \prime} \mathrm{S}\right.$ e $\left.40^{\circ} 20^{\prime} 16^{\prime \prime} \mathrm{W}\right)$ e Anchieta $\left(20^{\circ} 48^{\prime} 21^{\prime \prime} \mathrm{S}\right.$ e $\left.40^{\circ} 20^{\prime} 16^{\prime \prime} \mathrm{W}\right)$, localizados, respectivamente, no litoral norte, centro e sul do Estado do Espírito Santo. Em São Mateus, a coleta foi realizada no manguezal localizado em Campo Grande de Barra Nova; em Vitória, no bairro de São Pedro; e em Anchieta, na Rodovia do Sol, próxima à Vila dos Pescadores.

Foram coletados aleatoriamente um total de 172 indivíduos machos, sendo 62 espécimes provenientes de São Mateus, 60 de Vitória e 50 de Anchieta. A coleta dos indivíduos foi realizada por catadores de caranguejo de cada região, no modo tradicional manual.

Para a análise fenotípica, as características estudadas foram: largura cefalotorácica (LC), largura do $5^{\circ}$ somito abdominal (LSA), tamanho do quelípodo de defesa (TQ) e tamanho da quela (TQA).

As análises estatísticas foram feitas utilizando-se o programa SAS (1996), que emprega o modelo descrito a seguir:

$$
y_{i j k}=\mu_{k}+L_{i k}+e_{i j k}
$$

Onde:

$y_{i j k}=$ valor observado da característica $\mathrm{k}$ na repetição j do local i;

$\mu_{k}=$ média da característica $\mathrm{k}$;

$L_{i k}=$ efeito do local de coleta i na característica $\mathrm{k}$;

$\mathrm{e}_{\mathrm{ijk}}=$ efeito do erro aleatório associado à observação;

$\mathrm{Na}$ MANOVA, foram obtidas matrizes de soma de quadrados e produtos de dimensões quatro por quatro (número de características), sendo $\mathrm{A}, \mathrm{H}$ e $\mathrm{E}$ as matrizes de soma de quadrados e produtos totais, do local (tratamentos) e residuais, respectivamente. Sendo que a hipótese testada pela MANOVA é a de igualdade dos vetores de média:

$H_{0}:\left[\begin{array}{l}\mu_{11} \\ \mu_{12} \\ \mu_{13} \\ \mu_{14}\end{array}\right]=\left[\begin{array}{l}\mu_{21} \\ \mu_{22} \\ \mu_{23} \\ \mu_{24}\end{array}\right]=\left[\begin{array}{l}\mu_{31} \\ \mu_{32} \\ \mu_{33} \\ \mu_{34}\end{array}\right]=\left[\begin{array}{l}0 \\ 0 \\ 0 \\ 0\end{array}\right] \quad$ ou, $H_{0}: \underset{\sim 1}{L}=\underset{\sim 2}{L}=\underset{\sim}{L}$

Para testar essa hipótese, foi utilizado o teste de Roy, que consiste na determinação das raízes características (autovalores) da equação:

$$
\left|E^{-1} H-\lambda \|\right|=0 \quad \text { ou } \quad|H-\lambda E|=0
$$

R. Ci. méd. biol., Salvador, v. 4, n. 1, p. 15-23, jan./abr. 2005 
Sendo $\lambda_{\text {máx }} \mathrm{O}$ maior autovalor de $E^{-1} \mathrm{H}$ define-se a estatística $\theta_{0}=\frac{\lambda_{\text {máx }}}{1+\lambda_{\text {máx }}}$.

O valor assim obtido é comparado com o limite de significância fornecido em ábacos e tabelas encontrados, por exemplo, nos livros de Morrison (1967) e Harris (1975).

Os parâmetros da distribuição de sob a hipótese de nulidade são:

$$
\begin{aligned}
& \mathrm{s}=\operatorname{mín}(\mathrm{k}, \mathrm{q}) \\
& \mathrm{m}^{\prime}=(|\mathrm{k}-\mathrm{q}|-1) \\
& \mathrm{n}^{\prime}=1 / 2\left(\mathrm{n}_{\mathrm{e}}-\mathrm{k}-1\right) \\
& \text { em que } \\
& \mathrm{k}=\text { número de características analisadas; } \\
& \mathrm{q}=\text { número de graus de liberdade associ- }
\end{aligned}
$$
ado à matriz $\mathrm{H}$;

$\mathrm{n}_{\mathrm{e}}=$ número de graus de liberdade associado à matriz $\mathrm{E}$.

Rejeita-se $\mathrm{H}_{\mathrm{o}}$ ao nível de significância $\alpha$ se $\theta_{0}>\theta\left(\alpha, s, m^{\prime}, n^{\prime}\right)$.

Um resultado significativo na MANOVA pode ser interpretado como: existe, pelo menos, uma média das características consideradas que diferem de outra pelo teste de Roy; portanto, existe diferença entre os vetores de média.

Assim, para uma variável $\mathrm{k}$, para testar um contraste entre as médias, calculou-se a diferença mínima significativa, segundo Roy e Bose dada por:

$$
\text { DMS }=\sqrt{\frac{\theta_{\alpha}}{1-\theta_{\alpha}} \frac{\mathrm{SQR}_{\mathrm{r}}}{\overline{\mathrm{r}}} \sum_{\mathrm{i}=1}^{3} \mathrm{c}_{\mathrm{i}}^{2}}
$$

em que:

valor crítico de Roy, ao nível a de probabilidade com parâmetros $s$, m' e n';

$\mathrm{SQR}_{\mathrm{r}}=$ soma de quadrados do resíduo para a variável $\mathrm{k}$;

$\overline{\mathbf{r}} \mathrm{r}=$ aproximação do número de repetições utilizando-se a média harmônica;

Devido à necessidade de verificar se existe diferença entre os locais (tratamentos), utilizando-se uma estrutura multivariada, foi utilizada a função discriminante linear de Fisher (FDF). A função discriminante linear de Fisher é uma combinação linear das variáveis do tipo:

$$
\mathrm{Z}=\mathrm{a} \cdot \mathrm{LC}+\mathrm{b} \cdot \mathrm{TQ}+\mathrm{c} \cdot \mathrm{TQA}+\mathrm{d} \cdot \mathrm{LSA}
$$

Dada a análise de variância multivariada relativa às quatro variáveis consideradas, foram determinados os coeficientes da FDF por meio do seguinte procedimento:

1. Determinaram-se os autovalores da matriz $\mathrm{E}^{-1} \mathrm{H}$.

2. A partir do maior autovalor, determinou-se um autovetor.

3. Determinou-se o autovetor normalizado.

$$
\mathbf{v}_{\sim}^{\prime} E v=1
$$

Os coeficientes da FDF já aplicada aos dados de observaçóes reduzem o espaço tetradimensional a um espaço unidimensional. A partir desse ponto, realizou-se uma ANOVA em que a hipótese testada foi de igualdade dos locais (tratamentos).

$$
\mathrm{H}_{0}: \mathrm{L}_{1}=\mathrm{L}_{2}=\mathrm{L}_{3}
$$

Os coeficientes de ponderação das características foram obtidos pela solução dos sistemas

$$
\begin{gathered}
\left|H-\lambda_{j} E\right|=0 \\
\left(H-\lambda_{\mathrm{j}} \mathrm{E}\right) \underset{\sim \mathrm{j}}{\underset{\mathbf{v}}{\mathbf{n}}}=\mathbf{0}
\end{gathered}
$$

em que

$\mathrm{H}=$ matriz de variâncias e covariâncias entre os locais (tratamentos);

$\lambda_{\mathrm{j}}=\mathrm{j}$-ésimo autovalor;

$\mathrm{E}=$ matriz de variâncias e covariâncias residuais; e

$\underset{\sim j}{\mathrm{~V}}$ = autovetor associado a cada autovalor.

Foi realizada a análise de agrupamento segundo o método de Tocher, com base nas medidas de dissimilaridade da distância generalizada de Mahalanobis, denominada $\mathrm{D}^{2}$, que é estimada por:

$$
D_{i i^{\prime}}^{2}=\sum_{j}\left(Y_{i j}-Y_{i^{\prime} j}\right)^{2}
$$

\section{RESULTADOS}

Observaram-se diferenças significativas $(\mathrm{p}<0,01)$ entre os vetores de médias das 
populações pelo teste do maior autovalor de Roy. Os resultados do teste de Roy e Bose para comparação entre os vetores de médias são apresentados na Tabela 1, indicando que há diferença entre os valores fenotípicos da população.

Na Tabela 1 também foi observado que a média da largura cefalotorácica (LC) apresentouse mais elevada em Anchieta, não diferindo significativamente entre Vitória e São Mateus.

As características tamanho do quelípodo (TQ) e tamanho da quela (TQA) apresentaram médias mais elevadas em Anchieta, seguida por São Mateus e Vitória, respectivamente. A característica largura do $5^{\circ}$ somito abdominal (LSA) apresentou maiores médias em Vitória e Anchieta, não diferindo significativamente. São Mateus e Anchieta não apresentaram diferenças significativas com relação a essa característica.

A primeira variável canônica explicou $92,37 \%$ da variação observada (TABELA 2), sendo que, nesse estudo, foram obtidas duas variáveis canônicas. A característica LSA

Tabela 1 - Médias para os três locais pelo teste de Roy

\begin{tabular}{lccccc}
\hline Local & N & LC & TQ & TQA & LSA \\
\hline Vitória & 60 & $6,9317^{\mathrm{a}}$ & $9,7800^{\mathrm{a}}$ & $5,3017^{\mathrm{a}}$ & $1,1350^{\mathrm{a}}$ \\
São Mateus & 62 & $7,2597^{\mathrm{a}}$ & $11,7048^{\mathrm{b}}$ & $6,3790^{\mathrm{b}}$ & $1,0403^{\mathrm{b}}$ \\
Anchieta & 50 & $7,8333^{\mathrm{b}}$ & $13,1833^{\mathrm{c}}$ & $7,1250^{\mathrm{c}}$ & $1,0958^{\mathrm{ab}}$ \\
\hline
\end{tabular}

Notas: $\mathrm{N}$ = número de observaçôes; $\mathrm{LC}=$ largura cefalotorácica $(\mathrm{cm}) ; \mathrm{TQ}=$ tamanho do quelípodo de defesa $(\mathrm{cm}) ; \mathrm{TQA}=$ tamanho da quela $(\mathrm{cm})$; LSA $=$ largura do $5^{\circ}$ somito abdominal $(\mathrm{cm})$. As médias seguidas pela mesma letra, em cada coluna, não diferem estatisticamente pelo teste de Roy, a 5\% de significância.

Tabela 2 - Variáveis Canônicas (VCi) e autovetores (coeficientes) associados aos autovalores obtidos da análise das características para as três localidades

\begin{tabular}{|c|c|c|c|c|c|}
\hline $\mathrm{VCi}$ & Porcentagem & $\mathrm{LC}$ & TQ & TQA & LSA \\
\hline $\mathrm{VCl}$ & 92,37 & 0,07177869 & 0,05059592 & $-0,01892223$ & $-0,24782811$ \\
\hline $\mathrm{VC} 2$ & 7,63 & 0,06602431 & $-0,01430201$ & 0,01003456 & 0,54872410 \\
\hline
\end{tabular}

apresentou maior valor para a segunda variável canônica, indicando, portanto, que essa característica pouco contribui para a distinção das populaçôes e a avaliação da diversidade, podendo ser descartada das análises.

Os escores da primeira variável canônica foram usados para obter a seguinte função discriminante linear de Fisher, a partir da qual foram obtidos os escores representados na Tabela 3 , permitindo reunir as informaçôes das quatro variáveis em um único escore, o que auxilia a interpretação.

$$
\begin{aligned}
& \mathrm{FDF}=0,0717^{*} \mathrm{LC}+0,0505^{*} \mathrm{TQ}+ \\
& 0,0189^{*} \mathrm{TQA}+0,2478^{*} \mathrm{LSA}
\end{aligned}
$$

Tabela 3 - Função discriminante linear de fisher (FDF) com base na primeira variável canônica.

\begin{tabular}{lll}
\hline Local & $\mathrm{N}$ & Escores \\
\hline Vitória & 60 & $0,6108^{\mathrm{a}}$ \\
São Mateus & 62 & $0,7348^{\mathrm{b}}$ \\
Anchieta & 50 & $0,8255^{\mathrm{c}}$ \\
\hline DMS $^{*}$ & & 0,0591
\end{tabular}

Notas: $\mathrm{N}$ = número de observaçôes; DMS $^{*}=$ diferença mínima significativa. As médias seguidas pela mesma letra não diferem estatisticamente.

De acordo com os escores obtidos na função discriminante linear de Fisher (acima), as três localidades estão distantes umas das outras, de modo que essa distância é proporcional ao grau de dissimilaridade entre as populações.

Com base na distância de Mahalanobis, São Mateus encontra-se mais próximo a Anchieta, e Vitória apresenta-se mais distante dos dois locais (TABELA 4).

Tabela 4- Distância de Mahalanobis entre os locais analisados.

\begin{tabular}{lcc}
\hline Local & São Mateus & Anchieta \\
\hline Vitória & 2,9725 & 7,8053 \\
São Mateus & & 1,9234 \\
\hline
\end{tabular}

R. Ci. méd. biol., Salvador, v. 4, n. 1, p. 15-23, jan./abr. 2005 
A Figura 1 mostra a dispersão gráfica das três populações analisadas, de acordo com as médias canônicas e o agrupamento segundo o método de Tocher.

Pela técnica de agrupamento de Tocher, baseada na distância generalizada de Mahalanobis $\left(\mathrm{D}^{2}\right)$, São Mateus e Anchieta pertencem ao mesmo grupo e Vitória está num grupo isolado e, portanto, há similaridade entre São Mateus e Anchieta; ou seja, as características fenotípicas analisadas estão mais similares nessas duas localidades, mostrando proximidade entre elas. Esse resultado pode ser visualizado na Figura 1.

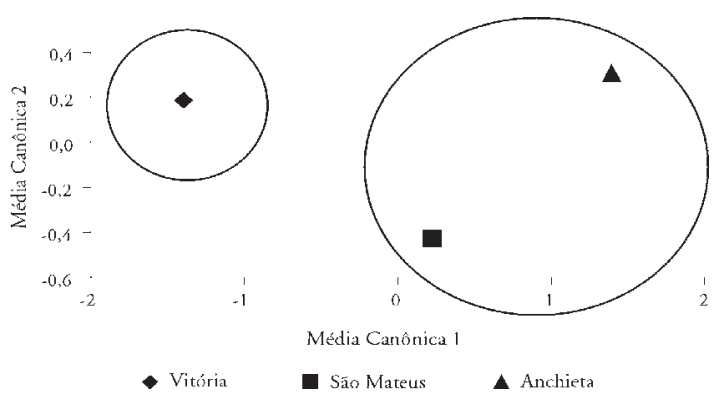

Figura 1 - Dispersão gráfica das localidades com relação as médias canônicas e a análise de agrupamento segundo método de Tocher

$\mathrm{Na}$ Tabela 5 são apresentados os valores dos coeficientes de correlação entre as características. Dentre as correlaçôes significativas estatisticamente, LC e TQ obtiveram um coeficiente de correlação intermediário $(0,5514)$, LC e TQA um coeficiente baixo $(0,3444)$ e TQ

Tabela 5 - Coeficiente de correlação entre as características

\begin{tabular}{rccc}
\hline & TQ & TQA & LSA \\
\hline LC & $0,5514^{* *}$ & $0,3444^{* *}$ & $0,4264^{* *}$ \\
TQ & & $0,8779^{* *}$ & $-0,0055^{\text {ns }}$ \\
TQA & & & $-0,1136^{\text {ns }}$ \\
\hline
\end{tabular}

Nota: ${ }^{* *}=$ significativo a $1 \% ;$ ns $=$ não-significativo; LC = largura cefalotorácica $(\mathrm{cm}) ; \mathrm{TQ}=$ tamanho do quelípodo de defesa $(\mathrm{cm}) ;$ TQA $=$ tamanho da quela $(\mathrm{cm}) ;$ LSA = largura do $5^{\circ}$ somito abdominal $(\mathrm{cm})$. e TQA uma alta correlação $(0,8779)$. As correlaçōes entre TQ e LSA, e TQA e LSA foram estatisticamente não-significativas.

A característica LC apresentou correlação com TQ e TQA, ou seja, quanto maior for LC maior será TQ e TQA. Por esse motivo, Anchieta apresentou as maiores médias para as três características.

\section{DISCUSSÃO}

Com base nos resultados obtidos, podese observar que a população de Vitória apresentou as menores médias para LC, TQ e TQA, provavelmente porque, nesse local, a pressão de coleta é muito elevada e, principalmente, desorganizada e não sustentável. Dessa maneira, ou foram exterminados grande parte dos indivíduos no tamanho correto para a coleta ( $6 \mathrm{~cm}$ de carapaça) e, por isso, estão sendo coletados indivíduos menores e (ou) está havendo uma seleção contra os animais maiores, de maneira que, no futuro, talvez isso venha comprometer a média de tamanho populacional dessa espécie em Vitória. Entrevistas realizadas pela equipe com as populações humanas que sobrevivem desse recurso corroboram os resultados, visto que a própria comunidade já percebe essa redução significativa do tamanho dos animais.

A captura dos indivíduos maiores poderia estar afetando a estrutura genética da população, dando maiores chances aos indivíduos sexualmente maduros com um menor tamanho corporal. Deve ser ressaltado que, em nenhuma população, foi observada LC equivalente a 9,6 $\mathrm{cm}$, que é o tamanho máximo atingido pelo macho, segundo Pinheiro e Fiscarelli (2001). Além do mais, tais seleções direcionais poderão comprometer em muito a variabilidade genética geral da espécie e, conseqüentemente, comprometer sua capacidade de adaptação a mudanças ambientais futuras.

Outro dado importante levantado por este trabalho foi o número de pessoas ("caranguejeiros") registradas para essa atividade. Em São Mateus, a quantidade de caranguejeiros 
registrados é de 324 pessoas, enquanto que, em Anchieta, há 38 caranguejeiros e, em Vitória, há aproximadamente 120 catadores. Dessa maneira, uma hipótese a se testar é que quanto maior a quantidade de pessoas extraindo o recurso, mais rápido o mesmo irá acabar. Tal fato sugere a adoção de medidas de exploração sustentáveis para essas comunidades de pessoas.

Os registros dos catadores de Vitória estão sendo reajustados pela Secretaria do Meio Ambiente desse município (SEMMAM), visto que o seu número aumentou desde o último registro, devido à quantidade de pessoas que não são caranguejeiros profissionais e que praticam essa atividade, muitas vezes de forma indiscriminada, o que pode estar contribuindo para o reduzido tamanho dos caranguejos de Vitória.

A alta correlação apresentada entre o tamanho do quelípodo e o tamanho da quela deve-se ao fato de essas características fazerem parte do mesmo membro, ou seja, no quelípodo, há uma pinça (ou garra) que recebe o nome de quela.

Para corrigir os possíveis erros associados à inclusão de indivíduos de diferentes classes de idades ou de sexos diferentes, os dados podem ser submetidos a uma Análise Discriminante Independente do Tamanho (ADIT), após o conhecimento dos padrôes alométricos das populações em estudo, o que é possível com a realização da Análise por componentes principais
(ACP) (REIS; PESSÔA; STRAUS, 1990; PERES-NETO, 1995). No entanto, esses estudos não foram realizados no presente trabalho. Uma outra metodologia a ser testada é a aplicação de marcadores moleculares neutros nessas populações, para confrontar os dados aqui obtidos, bem como para aumentar o conhecimento da variabilidade genética dessa espécie.

\section{CONCLUSÃO}

Observou-se diferença entre as três populações com base nas características analisadas, sendo que São Mateus e Anchieta possuem certo grau de similaridade, e Vitória está mais distante.

A falta de relação entre as partes corporais mostra que existe variação de uma característica em relação às outras, dentro de todo conjunto amostrado, e essas diferenças, se não forem devidas à alometria e às diferentes faixas etárias, geraram o padrão segregacional das populaçôes estudadas.

Não se pode inferir se essa diferença está diretamente relacionada com o genótipo da espécie estudada, pois as características mensuradas foram obtidas a partir de indivíduos sem padronização de idade, e não há estudos sobre o crescimento alométrico da espécie.

\title{
Morphometric analysis of mangrove crab (Ucides Cordatus L.) (Crustacea - Decapoda) populations in Espirito Santo mangroves coastline
}

\begin{abstract}
The mangrove crab (Ucides cordatus) is a crustacean (Decapoda: Brachyura) of great cultural, biological and commercial value in all Espirito Santo. However, there is still a great lack of data regarding the mangrove crab. In order to minimize this situation, the present work carried through a study on the population divergence of Ucides cordatus in Espirito Santo mangroves. Four morphometric features have been analysed in 172 male individuals, from São Mateus $(N=62)$, from Vitória $(N=60)$ and from Anchieta $(N=50)$. Meaningful difference between the three populations was observed $(p<0,01)$ by the Multivaried Analysis. The greatest degree of similarity was found between São Mateus (North) and Anchieta (South) so they had been classified in a single group. Vitória (Center) was enclosed in another group, evidencing divergence between this population and the other. This results suggests differents pressure by
\end{abstract}


selection in the interior populations in relation with de capital populations, which may be connected with a greater human action on the capital populations

Keywords: U. cordatus - genetic divergence; animal conservation; MANOVA.

\section{REFERÊNCIAS}

ALCANTRA-FILHO, P. Diversificação intraespecífica do caranguejo-uçá, Ucides cordatus cordatus (Linnaeus, 1763) (Crustácea - Decapoda - Brachyura), entre os manguezais dos Rios Pará, Estado do Pará e Pomonga, estado de Sergipe (Brasil): $00^{\circ} 41^{\prime} \mathrm{S}-10^{\circ} 40^{\prime} \mathrm{S}$. 1982. 61f. Tese (Doutorado em Ciências)Programa de Pós-Graduação em Ciências, Universidade de São Paulo, São Paulo, 1982.

ARAÚJO, C.F. Avaliação do desempenho de linhas de frango de corte em desenvolvimento na UFV e de seus cruzamentos em relação à duas marcas comerciais. 1995. 108f. Dissertação ( Mestrado em Genética e Melhoramento Animal)-Programa de Pós-Graduação em Genética e Melhoramento Animal, Universidade Federal de Viçosa, Viçosa, 1995.

CARMO, T.M.S. Os manguezais ao norte da baía de Vitória, ES. In: SIMPÓSIO SOBRE ECOSSISTEMAS DA COSTA SUL E SUDESTE BRASILEIRA, 1., 1987, São Paulo. Síntese dos conhecimentos. São Paulo: ACIESP, 1987. v.1, p.173-193.

CARNEIRO, P.L.S.et al. Estudo da divergência genética entre linhagens de matrizes de frangos de corte por meio de análise multivariada. Arq. Bras. Med. Vet. Zootec., Belo Horizonte, v.54, n.1, p.75-83, 2002.

CRUZ, C.D.; REGAZZI, A.J. Modelos biométricos aplicados ao melhoramento genético. Viçosa: Universidade Federal de Viçosa, 1997.

DIELE, K. Life history and population structure of exploited mangrove crab Ucides cordatus (L.) (Decapoda: Brachyura) in the Caeté estuary, north Brazil. Bremen: Zentrum für Marine Tropenökologie, 2000. (ZMT Contributions, 9)
FONSECA, R. da. et al. Estudo da divergência genética entre raças suínas utilizando técnicas de análise multivariada. Arq. Bras. Med. Vet. Zootec., Belo Horizonte, v.52, n.4, p.403-409, 2000.

FREIRE, A.S. Dispersão larval do caranguejo do mangue Ucides cordatus (Linnaeus, 1763) em manguezais da Baía do Paranaguá, Paraná. 1998. 67f. Tese (Doutorado em Ciências)Programa de Pós-Graduação em Ciências, Universidade de São Paulo, São Paulo, 1998.

FUTUYMA, D.J. Biologia evolutiva. 2.ed. São Paulo: FUNPEC, 2002.

GRIFFTHIS, A.J.F. et al. Introdução à genética. 7.ed. Rio de Janeiro: Guanabara Koogan, 2002.

HARRIS, R.J. A primer of multivariate statistics. New York: Academic Press, 1975.

MATIOLI, S.R. Biologia molecular e evolução. São Paulo: Holos, 2001.

MORRISON, D.F. Multivariate statistical methods. New York: McGraw-Hill, 1967.

NUNES, A.G.N. Os argonautas do mangue: uma etnografia visual dos caranguejeiros do município de Vitória - ES. 1998. 207f. Dissertação (Mestrado em Multimeios)Programa de Pós-Graduação em Multimeios, Universidade de Campinas, Campinas, 1998.

PAIVA, S.R. Influência de obstáculos naturais na divergência de populações de Astyanax bimaculatus na bacia do Rio Doce - MG. Dissertação (Mestrado em Genética e Melhoramento Animal)-Programa de PósGraduação em Genética e Melhoramento Animal, Universidade Federal de Viçosa, Viçosa, 2001. 
PERES-NETO, P.R. Introdução a análises morfométricas. In: PERES-NETO, P.R.; VALENTIN, J.L.; FERNANDEZ, F. (Ed.). Tópicos em tratamento de dados biológicos. Rio de Janeiro: Universidade Federal do Rio de Janeiro, Instituto de Biologia, Programa de PósGraduação em Ecologia,1995. p.57-89. (Oecologia Brasiliensis, v.2)

PINHEIRO, M.A.A.; FISCARELLI, A.G. Manual de apoio à fiscalização do caranguejouçá (Ucides cordatus). Itajaí: CEPSUL/IBAMA, 2001.

PRATES-JR, P. et al. Diversidade genética do Lobo-Guará, Chrysocyon brachyurus (Illiger, 1811), com base na variabilidade do DNA mitocondrial. In: CONGRESSO NACIONAL DE GENÉTICA, 49., 2003, São Paulo. Anais... São Paulo: Sociedade Brasileira de Genética, 2003. 1CD-ROM.

REIS, S.F.; PESSÔA, L.M.; STRAUS, R.E. Application of size-free canonical discriminant analysis to studies of geographic differentiation. R. Bras. Genética, São Paulo, v.13, n.3, p.509520, 1990.
RODRIGUES, M.D. Desenvolvimento pósembrionário de Ucides cordatus (Linnaeus, 1763) (crustácea, decapoda, gecarcinidae). 1982. 85f. Dissertação (Mestrado em Zoologia de Invertebrados)-Programa de Pós-Graduação em Zoologia de Invertebrados, Universidade Estadual Paulista "Júlio de Mesquita Filho", São Paulo, 1982.

SAS/STAT user's guide: version $6.4^{\text {th }}$ ed. Cary: SAS INSTITUTE, 1990.

TAVARES, E.S.; MIYAKI, C.Y. Utilização de sequências parciais de $D_{N A} 12 S$ e $16 S$ em reconstruções filogenéticas entre gêneros de psitacídeos (aves, psittaciformes). In: CONGRESSO NACIONAL DE GENÉTICA, 49., 2003, São Paulo. Anais... São Paulo: Sociedade Brasileira de Genética, 2003. 1 CDROM.

VIANNA, J.A.; MARMONTEL, M.; SANTOS, F.R. Filogeografia e diversidade genética de Boto Rosa (Inia geoffrensis). In: CONGRESSO NACIONAL DE GENÉTICA, 49., 2003, São Paulo. Anais... São Paulo: Sociedade Brasileira de Genética, 2003. 1 CDROM.

Recebido em / Received: 02/03/2005 Aceito em / Accepted: 20/04/2005 